\title{
Rationally respond to post-vaccination adverse events
}

\author{
WU Ting, ZHANG Jun \& XIA NingShao* \\ School of Public Health, Xiamen University, National Institute of Diagnostics and Vaccine Development in Infectious Diseases, State Key \\ Laboratory of Molecular Vaccinology and Molecular Diagnostics, Fujian 361102, China
}

Received January 3, 2014; accepted January 28, 2014; published online February 12, 2014

Citation: Wu T, Zhang J, Xia NS. Rationally respond to post-vaccination adverse events. Sci China Life Sci, 2014, 57: 557-560, doi: $10.1007 / \mathrm{s} 11427-014-4623-4$

Vaccination is one of the most powerful tools to extend the average lifespan of humans. Vaccination has successfully eradicated smallpox and significantly reduced the morbidity and mortality of several infectious diseases, such as poliomyelitis [1]. It is currently one of the most effective measures to control infectious diseases [1]. The health benefits brought by widespread vaccination are beyond doubt, whether for public health or individuals.

However, the development of vaccination has also been accompanied by tragedy. In the late 19th century, the rabies vaccine that was produced by nerve tissue cells prevented a large number of deaths, but epilepsy, paralysis and/or coma appeared in one of every 230 persons vaccinated [2]. In 1942, hundreds of thousands of American soldiers were vaccinated with the yellow fever vaccine, which contained human serum. Some of the serum was from hepatitis B virus carriers, and 330000 soldiers were infected; 50000 developed with acute hepatitis and 62 died [3]. In 1955, an American company produced the inactivated polio vaccine, but the inactivation was not sufficient. Among the 120000 children who were vaccinated with this lot of vaccine, there were 40000 cases of mild poliomyelitis, 200 cases of permanent paralysis, and 10 deaths [4].

No medical intervention is absolutely safe. Even if a population with the required health status receives a vaccine of reliable quality according to the normal procedures, rare but serious adverse reactions may occur. For example, the attenuated oral polio vaccine can cause paralytic poliomyelitis [5]; the whole-cell pertussis vaccine can cause acute

*Corresponding author (email: nsxia@xmu.edu.cn) encephalitis [6]; the rubella vaccine can cause acute arthritis [7]; some influenza vaccines can cause Guillain-Barre syndrome [8]; and the measles, mumps and rubella (MMR) vaccine containing gelatin can cause severe allergic reactions [9].

The target of vaccination is a healthy population. While the tolerance of healthy people for adverse events after vaccination is significantly lower than that of the patients who need other medical intervention measures such as drugs and surgery. Therefore, the safety standards of vaccines are significantly higher than those of other medical intervention measures. The safety monitoring of vaccines begins at the pre-clinical development phase, and continues through registered clinical trials and after the product is brought to the market. Before a vaccine enters clinical trials, its safety must be verified in various animal models. If the vaccine may be used for women of childbearing age, the safety verification must include sufficient assessment during pregnancy and any impact on embryonic growth and development must also be assessed. In phase I-III clinical trials, vaccine safety and efficacy is usually assessed gradually in thousands, to tens of thousands, and $>100000$ volunteers. In phase II/III trials, usually a randomized controlled double-blind design is used, with volunteers randomly assigned to test and control groups. The test group is treated with vaccine, while the control group receives a placebo (blank adjuvant or irrelevant vaccine). Safety assessment includes observation of local reactions (such as pain, redness, swelling and induration) and systemic reactions (such as fever, allergic skin rash, fatigue, weakness and headache) within 30 days post vaccination. Safety assessment also includes 
the incidence and correlation with vaccination of all serious adverse events (such as hospitalization, death, permanent disability, and fetal malformation) throughout the clinical trial (usually 1-3 years). Usually the time relevance, biological plausibility, association specificity, strength of association, consistency of association, and other principles need to be considered when determining the correlation between vaccines and adverse events, which is a complex process.

Vaccine related serious adverse reactions are usually rare, occurred delayed, some particular subgroups may have higher risk of adverse reactions, and it is not possible to expand the clinical trial sample size indefinitely, thus they are difficult to be detected. Historically, the assessment of rare adverse reactions mainly depended on the establishment of a passive adverse event reporting system after the vaccine came on the market, and on some targeted epidemiological studies. Recently, more countries have established routine reporting systems for adverse events in phase IV clinical trials after the vaccine has come to market. These measures significantly improve our ability to detect serious vaccine adverse reactions, as well as any defects in the vaccine lot or its manufacture. However, some systemic defects that cannot be overcome still exist in this system. For example, the baseline incidence of some serious adverse events is unknown, and the baseline disease risk differs according to the socioeconomic status of the vaccinated and unvaccinated populations. These differences make it difficult to judge whether the vaccinated population really has a higher risk of adverse events.

Usually, the national vaccine administrator will decide on how the vaccine should be used after scientific assessment of the adverse reactions after vaccination. The existing rotavirus vaccine on the European and American markets may cause infant intussusception after the first dose, with an incidence of about one to two per hundred thousand. However, compared with hospitalization and death caused by vaccine-preventable rotavirus infection, the benefits outweigh the risks, thus, these two vaccines are still in use [10].

Domestic and international experience show that (Figure 1) along with the increased rate of vaccination, most people have developed protective antibodies; susceptible populations have been reduced; and the spread of pathogenic microorganisms has become difficult, which resulted in a rapid decline in disease incidence. Because the relevant diseases are on the wane and vaccine adverse events start to gain more attention, then the willingness to receive vaccination is decreasing. This is resulting in a resurgence of the diseases and their incidence, much more people suffered from the disease. Having experienced the painful lessons, people have started to receive vaccination again and the vaccination rate has gradually recovered. If vaccination rates could be maintained for a certain period, the diseases in question could be eliminated. As a result, people would no longer need vaccination, and any adverse events caused by the disease or vaccination would be eliminated at the same time.

Several large-scale campaigns to boycott vaccines have occurred but they have all ended with resumption of vaccination at the expense of suffering from the epidemic diseases. From the 1970s to 1980 s, extensive vaccination with whole-cell pertussis reduced the incidence of pertussis to below 1/100000, which resulted in doubt about the need for vaccination [11]. A report that 36 infants developed neurological symptoms after vaccination dented expert and public confidence in the vaccine in the UK, and the vaccination rate decreased from $81 \%$ to $31 \%$ [11]. This resulted in an increase in the incidence of pertussis to $>100 / 100000$, with $>100000$ cases and 36 deaths. To gain people's confidence, the British government took active measures to re-evaluate the effect of vaccination. The incidence of pertussis returned to its previous low level after the vaccination rate increased to $93 \%[11,12]$. At the same time, Japan changed the diphtheria, tetanus and pertussis vaccine into the diphtheria and tetanus vaccine for the same reason. After two infants died as a result of vaccination with whole-cell pertussis in Japan, and the Ministry of Health stopped pertussis vaccination of infants. Consequently, the vaccination rate decreased from $80 \%$ in 1974 to $10 \%$ in 1976. In $1979,>13000$ cases of pertussis appeared and 41 people died [11]. In the UK, measles vaccine is another controversial issue. In 1998, Wakefield et al. published journal paper in The Lancet, which claimed a connection between MMR vaccination and autism in children [13]. As a result of media publicity, MMR vaccination rate decreased from $92 \%$ in 1995-1996 to 80\% in 2003-2004 [14]. Since 1999, the UK has experienced a continuous outbreak of measles [15]. In the meantime, the scientific community carried out a lot of research to verify the connection between the MMR vaccine and autism in children. In 2003, sufficient scientific data proved that MMR vaccine has no connection with autism in children [16,17]. After that, the MMR vaccination rate rose gradually, reaching $90 \%$ in 2010; its highest for nearly 13 years. However, this is still lower than $95 \%$ measles vaccination rate recommended by the World Health Organization. In 2010, Wakefield's paper was revoked by The Lancet.

Hepatitis B vaccine is a mature agent. In China, the use of hepatitis B vaccine began in 1986 and it was included in the childhood immunization program in 1992. As a result, China has made great progress in controlling hepatitis B virus (HBV) infection. From 1992 to 2006, the seroprevalence of hepatitis B surface antigen ( $\mathrm{HBsAg}$ ) in people aged 1-59 years decreased from $9.75 \%$ to $7.20 \%$ [18]. There was also a reduction of 16-20 million $\mathrm{HBsAg}$ carriers and 2.8-3.5 million deaths from HBV infection. In those aged $<5$ years, the HBsAg positivity was only $1.0 \%$, a decrease of $90 \%$. Newborn infants and infants have received hepatitis $B$ vaccination worldwide, which has proved the safety of the vaccine. It has been reported that hepatitis $B$ vaccine 


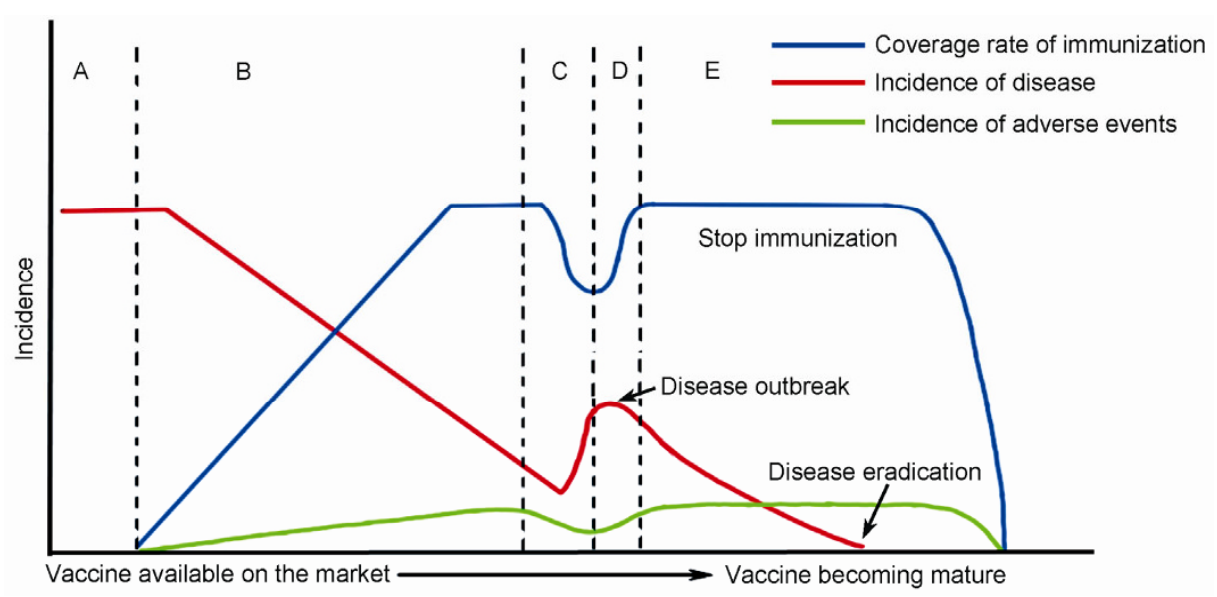

Figure 1 Promotion of vaccination programs and changes in disease (modified from [1]). A, Before vaccination. B, Coverage rate significantly increased after vaccine appeared on the market. C, Period of fluctuation in public willingness for vaccination. D, Vaccination willingness recovery period. E, Pathogens and relevant disease elimination period.

may be related to multiple sclerosis (MS) and other autoimmune diseases in overseas countries. American and European researchers have conducted a case-control study [19] and a retrospective analysis of MS cases in a European database [20]. They have found that hepatitis B vaccine does not increase the risk of multiple sclerosis. In China, the public health emergency events surveillance system from January 2006 to March 2007 showed that there were 10 deaths after hepatitis B vaccination. In two cases, it could not be ruled out acute anaphylactic shock after vaccination, and in the remaining eight cases, the primary causes of death had nothing to do with hepatitis B vaccine. According to the historical data, the safety and effectiveness of hepatitis $B$ vaccine are beyond doubt.

Recently, many areas of China have reported deaths after hepatitis B vaccination, which have raised media concern. Were these deaths a coincidence, or were they caused by quality problems with certain lots of vaccines or some manufacturers, or the hepatitis B vaccine itself? We are presently awaiting the results of further investigation. Combined with the earlier polio vaccine adverse events, which have attracted extensive societal concern, we should pay special attention, be vigilant about the resurgence of anti-vaccine campaigns, and avoid repeating in China the historical mistakes in other countries. Most cases of chronic hepatitis B in China are caused by mother-to-child transmission or childhood infection. If the Chinese neonatal hepatitis B vaccination rate decreased from $90 \%$ to $70 \%$, it would mean that 3.2 million among 16 million neonates would face the risk of HBV infection each year. When calculated in accordance with the HBsAg seroprevalence of Chinese women of childbearing age $(7 \%)$, the perinatal transmission rate of the HBsAg-positive mothers (40\%) and hepatitis B vaccine protection rate of newborns born to HBsAg-positive mothers $(90 \%)$, another 40000 newborns $(16000000 \times 7 \% \times 40 \% \times$ $90 \% \times 10 \%=40000)$ will be infected each year if vaccination rate falls by $10 \%$. Among the 40000 infected newborn infants, 90\% (36000) will develop chronic infection and $25 \%$ (10000) chronic liver disease (including cirrhosis and hepatocellular carcinoma). Therefore, we should rationally respond to vaccine adverse events, actively establish the correlation and causes of adverse events, and take appropriate response measures to minimize the damage.

1 Stanley A, Plotkin WO, Offit PA. Vaccines. 6th ed. New York: Elsevier, 2013. 1464-1480

2 Bahri F, Letaief A, Ernez M, Elouni J, Chekir T, Ben Ammou S, Jemni L. Neurological complications in adults following rabies vaccine prepared from animal brains. Presse Med, 1996, 25: 491-493

3 Seeff LB, Beebe GW, Hoofnagle JH, Norman JE, Buskell-Bales Z, Waggoner JG, Kaplowitz N, Koff RS, Petrini JL Jr, Schiff ER, et al. A serologic follow-up of the 1942 epidemic of post-vaccination hepatitis in the United States Army. N Engl J Med, 1987, 316: 965-970

4 Offit PA. The Cutter Incident: How America's First Polio Vaccine Led to the Growing Vaccine Crisis. New Haven: Yale University Press, 2005

5 Poliomyelitis prevention in the United States: introduction of a sequential vaccination schedule of inactivated poliovirus vaccine followed by oral poliovirus vaccine. Recommendations of the Advisory Committee on Immunization Practices (ACIP). MMWR Recommendations and reports: Morbidity and mortality weekly report Recommendations and reports/Centers for Disease Control. 1997, 46: 1-25

6 Miller D, Wadsworth J, Diamond J, Ross E. Pertussis vaccine and whooping cough as risk factors in acute neurological illness and death in young children. Dev Biol Stand, 1985, 61: 389-394

7 Peltola $\mathrm{H}$, Heinonen OP. Frequency of true adverse reactions to measles-mumps-rubella vaccine. A double-blind placebo-controlled trial in twins. Lancet, 1986, 1: 939-942

8 Schonberger LB, Bregman DJ, Sullivan-Bolyai JZ, Keenlyside RA, Ziegler DW, Retailliau HF, Eddins DL, Bryan JA. Guillain-Barre syndrome following vaccination in the National Influenza Immunization Program, United States, 1976-1977. Am J Epidemiol, 1979, 110: $105-123$

9 Kelso JM, Jones RT, Yunginger JW. Anaphylaxis to measles, mumps, and rubella vaccine mediated by IgE to gelatin. J Alergy Clin Immunol, 1993, 91: 867-872

10 Rotavirus vaccines: WHO position paper-January 2013. Wkly Epidemiol Rec, 2013, 88: 49-64 
11 Gangarosa EJ, Galazka AM, Wolfe CR, Phillips LM, Gangarosa RE, Miller E, Chen RT. Impact of anti-vaccine movements on pertussis control: the untold story. Lancet, 1998, 351: 356-361

12 Baker JP. The pertussis vaccine controversy in Great Britain, 1974-1986. Vaccine, 2003, 21: 4003-4010

13 Sabra A, Bellanti JA, Colón AR. Ileal-lymphoid-nodular hyperplasia, non-specific colitis, and pervasive developmental disorder in children. Lancet, 1998, 351: 637-641

14 Health Protection Agency. Annual COVER report: 2005/06 Summary of trends in vaccination coverage in the UK. Available: http://www.hpa.org.uk/web/HPAwebFile/HPAweb_C/119494736731 6

15 Jansen VA, Stollenwerk N, Jensen HJ, Ramsay ME, Edmunds WJ, Rhodes CJ. Measles outbreaks in a population with declining vaccine uptake. Science, 2003, 301: 804

16 Madsen KM, Hviid A, Vestergaard M, Schendel D, Wohlfahrt J, Thorsen P, Olsen J, Melbye M. A population-based study of measles, mumps, and rubella vaccination and autism. N Engl J Med, 2002, 347: $1477-1482$
17 Taylor B, Miller E, Farrington CP, Petropoulos MC, Favot-Mayaud I, Li J, Waight PA. Autism and measles, mumps, and rubella vaccine: no epidemiological evidence for a causal association. Lancet, 1999, 353: 2026-2029

18 Cui FQ. Epidemiology of seroprevalence of hepatitis B in Chinese population-the hepatitis $B$ vaccine immunization decreased the hepatitis B virus infection rate (in Chinese). Zhong Guo Yi Miao He Mian Yi, 2010, 16: 341-353

19 Mikaeloff Y, Caridade G, Rossier M, Suissa S, Tardieu M. Hepatitis $B$ vaccination and the risk of multiple sclerosis. N Engl J Med, 2001, 344: 327-332

20 Confavreux C, Suissa S, Saddier P, Bourdès V, Vukusic S; Vaccines in Multiple Sclerosis Study Group. Vaccinations and the risk of relapse in multiple sclerosis. Vaccines in Multiple Sclerosis Study Group. N Engl J Med, 2001, 344: 319-326

21 Wang FZ, Cui FQ, Liu DW. Analysis on the adverse events following immunization of 10 infants death after hepatitis B vaccination (in Chinese). Zhong Guo Yi Miao He Mian Yi, 2009, 15: 52-57

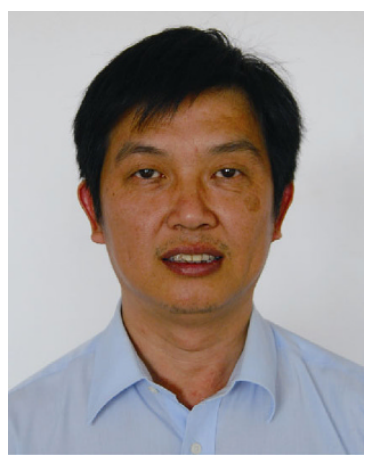

\section{Biographical Sketch}

Xia NingShao is Director of NIDVD, supervisor of State Key Laboratory of Molecular Vaccinology and Molecular Diagnostics, President of the School of Public Health of Xiamen University, doctoral supervisor, distinguished Minjiang Scholar and member of the "Ten Thousand Talent Plan" launched by the Organization Department of the Central Committee of the CPC. In addition, he holds a concurrent post as a part-time member of the 4th CSRC Growth Enterprise Board Public Offering Review Committee, president of "Infectious Disease Diagnostic Reagent Industrial Technology Innovation Strategic Alliance", a member of the major national science and technology special "Prevention and Treatment of AIDS and Virus Hepatitis" program (principle expert team) and head of the Hepatitis Team, member of the Medical Biotechnology Subject Matter Panel of Experts of National High Technology Research and Development Program and member of the Reviewing Expertise Group of the Department of Health Sciences of the National Natural Science Foundation. He mainly engages in biomedical research, and has successively committed himself to many national major/key subjects (e.g. National High Technology Research and Development Program of China), managed and developed the world's first hepatitis E vaccine, developed diagnostic products for AIDS, influenza, hand foot and mouth disease and hepatitis, won the second prize of the National Invention Awards, second prize of the National Science and Technology Progress Awards and Chinese Patent Gold Medal, was awarded 22 patents and 37 new drug/machinery certificates (one type I new drug, five EU certifications and one Taiwan certification), and has published more than 90 papers in The Lancet, Proc Natl Acad Sci USA and other SCI publications.

Open Access This article is distributed under the terms of the Creative Commons Attribution License which permits any use, distribution, and reproduction in any medium, provided the original author(s) and source are credited. 